\title{
VIOLENCIA ESCOLAR EN ESTUDIANTES DE EDUCACIÓN MEDIA
}

\author{
SCHOOL VIOLENCE IN HIGH SCHOOL STUDENTS \\ Daniela Baridon Chauvie \\ Universidad Internacional de La Rioja, España \\ Gema Martín Seoane \\ Universidad Complutense de Madrid, España
}

\begin{abstract}
Resumen: En este artículo se plantean los resultados de un estudio sobre la violencia escolar en centros de Educación Media de una ciudad del interior de Uruguay. Se presenta la percepción de 775 estudiantes de ambos sexos, de entre 12 y 19 años, de dos liceos y una escuela técnica de nivel secundario. El instrumento aplicado es el Cuestionario de Evaluación de la Violencia en la Escuela y el Ocio -CEVEO- (Díaz-Aguado, Martínez \& Martín, 2004) validado en España. Los resultados destacan la presencia de diversas formas de violencia entre iguales, así como carencias en la relación entre profesor y alumno. Este trabajo busca contribuir al conocimiento sobre la violencia escolar entre adolescentes, en los centros de Educación Media de Uruguay.
\end{abstract}

Palabras Clave: violencia escolar, bullying, convivencia escolar

\begin{abstract}
In this paper the results of a survey of middle school violence in a provincial town from Uruguay are presented. Data from 775 students of both sexes, aged 12-19, from two local high schools and a technical school were obtained. The School Violence and Leisure Assessment Questionnaire, which had been previously validated in Spain, was used (Díaz-Aguado, Martínez y Martín, 2004). The results reveal various forms of peer violence, as well as shortcomings in the relationship between teacher and student. This work aims to contribute to the knowledge about school violence among adolescents in middle education centers in Uruguay.
\end{abstract}

Keywords: school violence, bullying, school life

\section{Introducción}

La violencia entendida como "una violación o un arrebato al ser humano de algo que le es esencial como persona (integridad física, psíquica o moral, derechos, libertades...)" (como se citó en Torrego, 2006, p. 20) se presenta como una constante en la historia de la humanidad (Galtung,1998). Como fenómeno actual, no cabe duda que se expande a todo nivel, golpeando a las diferentes culturas, países, edades, etc. Torrego (2006) señala la ubicuidad de la violencia en nuestra sociedad actual, siendo el espacio educativo un ámbito más, para el desarrollo de la violencia. A tal punto que hoy en día la violencia escolar se ha convertido en un tema de potencial interés y es investigado en diversos contextos (O'Brien \& Bierman, 1988; Salmivalli, Lagerspetz, Björkqvist, Österman, Kaukianen, 1996; Smith, 1998; Hugh-Jones \& Smith, 1999; Sutton, Smith \& Swettenham, 1999; Pellegrini, 2002; Pikas, 2002; Shapiro, 2006). Si bien cabe señalar que la violencia escolar es tan antigua como la propia institución educativa (Pulido, Martín y Lucas, 2011), resulta importante destacar su vigencia, así como la necesidad urgente de intervenir de cara a erradicarla.

En América Latina el interés y conocimiento acerca de la convivencia y violencia escolar, puntualmente de la violencia entre iguales, se ha incrementado en las últimas décadas (Chaux \& Velásquez; 2008; Berger, 2009; Lisboa y Koller, 2009; Kornblit, Adaszko \& Di Leo, 2009). En Uruguay en particular, habrína confluido varias situaciones: por una parte los medios de comunicación evidenciaron diversas situaciones de violencia (vandalismo en muchos casos), que generaron alarma en la sociedad. Por otra parte la comunidad educativa fue tomando contacto con esta problemática estudiada en otros contextos y ambos hechos, se sumaron al interés de gran parte del profesorado e investigadores dedicados a la educación, de conocer lo que ocurre con las dinámicas de convivencia en los

Correspondencia: Daniela Baridon Chauvie. Universidad Internacional de La Rioja, España.

Correo Electrónico: daniela.baridon@unir.net 
centros y de cómo mejorar la calidad de vida en el espacio educativo. Actualmente contamos con relevantes investigaciones acerca de la violencia entre iguales y la convivencia escolar (Viscardi, 2004; Cajigas, Kahan et al., 2006; Pérez Algorta, 2006; Cajigas, Luzardo \&, Mungay, 2013; Cajigas, Luzardo \& Ugo, 2009; Giorgi, Kaplún \& Morás, 2012), interés que continúa creciendo en la comunidad educativa y al que espera contribuir este artículo de investigación.

Nos preocupa la violencia escolar, las concepciones que la legitiman y naturalizan en el espacio educativo, la invisibilidad de algunos sucesos violentos, las emociones de nuestros alumnos y de nosotros, los adultos. En este sentido entendemos que es necesario conocer en profundidad lo que ocurre para poder actuar sobre ello y trabajar en la construcción de un espacio escolar de convivencia sano y de calidad para todos. Para que la escuela sea el espacio por excelencia para la convivencia, al que está llamada ser, convirtiéndose en una herramienta a favor de la construcción de ciudadanos y de una sociedad más pacífica. Compartimos la idea de Chaux y Velásquez (2008) acerca de que, desde la escuela podemos promover el desarrollo de competencias en nuestros niños y adolescentes para tal fin. Se hace entonces necesaria una intervención eficaz en la detección y prevención de la violencia en los centros escolares.

Estos intereses y estas preocupaciones, asociadas a la posibilidad de profundizar en el tema a partir de estudios sobre convivencia y violencia escolar desarrollados en España, promovieron este trabajo con el fin de contribuir al acercamiento del conocimiento de lo que ocurre con el tema en el contexto uruguayo. Particularmente se investigó en tres centros de Educación Media de una ciudad del interior del país, con el objetivo principal de conocer la perspectiva de los adolescentes acerca de la incidencia de la violencia entre iguales en los tres centros existentes en dicha ciudad, con el fin último de ofrecer alternativas de intervención a la comunidad educativa de dichos centros (Baridon, 2010).

\section{Marco Teórico}

Las perspectivas actuales en el estudio de la violencia escolar reflejan la dificultad de, reflejan la dificultad de definir y delimitar con exactitud el término violencia, debido a lo versátil y ubicuo que es este fenómeno (Torrego \& Moreno, 2003; Torrego, 2007). Del Barrio, Martín, Almeida y Barrios (2003) señalan que la violencia hace referencia a: "todos los fenómenos de destrucción, fuerza, coerción, que ocurren en las relaciones, la sociedad 0 incluso la naturaleza" (p. 15).

Destacan que la violencia va más allá de las relaciones personales y se ejerce en el propio funcionamiento de la sociedad, lo que correspondería a la violencia estructural que menciona Galtung (1998). Díaz-Aguado (2004) señala que la violencia tiene causas múltiples y complejas; en tanto Fernández (1998) destaca la crueldad del ejercicio de la violencia, acción que daña tanto al que la recibe como a quien la ejerce.

La violencia no se puede justificar, menos aún en el ámbito educativo. Es sin duda un fenómeno social (Ortega, Del Rey \& Mora-Merchán, 2001) que se desencadena en la interacción del ser humano y su contexto, con lo cual se destaca la necesidad de analizarla desde una perspectiva evolutiva y ecológica (Bronfenbrenner, 1987). En este sentido se insiste en la relevancia de conocer la perspectiva de los adolescentes al respecto de la convivencia escolar en los centros a los que asisten, principalmente en lo que refiere a las relaciones y la violencia escolar entre iguales.

La violencia "toma múltiples expresiones y formas según el ámbito y actores que involucra" (Giorgi et al., 2012, p. 17). En el contexto escolar, ya en 1990 Hurrelmann destacaba la amplitud y complejidad de la noción de violencia escolar, señalando que el fenómeno involucra todo lo que causa dolor o lesiones tanto a nivel psíquico como físico a los demás, así como el daño a los objetos (Hurrelmann, 1990). La violencia escolar refiere a una "amplia gama de acciones que tienen por objeto producir daño y que alteran en mayor o menor cuantía el ámbito institucional" (Martínez-Otero, 2005, p. 35); con lo cual entendemos que involucra "cualquier comportamiento que viole la finalidad educativa de la escuela o el clima de respeto, o que ponga en peligro los intentos de la escuela para verse libre de agresiones contra personas o propiedades" (como se citó en Martín, 2008, p. 26), incluyendo conductas antisociales, agresiones, bullying (también llamado maltrato entre iguales, acoso u hostigamiento) y violencia propiamente dicha.

Si bien aquí no realizaremos un análisis conceptual exhaustivo, caben ciertas puntua- 
Violencia escolar en estudiantes de educación media

lizaciones. Por ejemplo, en torno al término agresión, definido en diversidad de trabajos (Parke \& Slaby, 1983; Coie \& Dodge, 1998, Del Barrio et al., 2003) y que en resumen destaca como un comportamiento intencionado y que busca un resultado o fin último, que es provocar un daño. El siguiente término a comentar, es bullying, término anglosajón acuñado desde los trabajos de Olweus (2004) y que implica una de las formas de violencia escolar por demás grave, pues hace referencia a situaciones en las que:"un alumno es agredido o se convierte en víctima cuando está expuesto, de forma repetida y durante un tiempo, a acciones negativas que lleva a cabo otro alumno o varios de ellos" (p. 25).

Entendiendo como acciones negativas a aquellas en que se le roba, se le pega, amenaza, ridiculiza en público (entre otras), y donde existe un abuso de poder desde quien ejerce la violencia sobre quién la recibe. Si bien el fenómeno del bullying es uno de los más estudiados a nivel internacional (Olweus, 2004; Salmivalli et al., 1996; Sutton et al., 1999; Hugh-Jones \& Smith, 1999; Pellegrini, 2002), no es la única forma de violencia existente en el ámbito educativo, como hemos señalado anteriormente.

Como aquí se señala, la violencia en el contexto escolar, presenta una tipología amplia y variada que constituye el fenómeno de violencia escolar. La investigación macro realizada (Baridon, 2010) se ha apoyado en diversas investigaciones del contexto nacional e internacional ya mencionadas, siendo la referencia principal el Programa de Prevención de la Violencia y Lucha contra la Exclusión desde la Adolescencia desarrollado en el contexto español por el equipo de investigación de María José Díaz-Aguado, y respetando la tipología allí presentada (DíazAguado, Martínez, \& Martín, 2004). Se contó con la participación de los estudiantes para realizar la primera parte de la investigación; asimismo se trabajó con los docentes y documentos del centro en fases posteriores, lo cual permitió triangular la información y obtener una visión más completa de la situación de dicho contexto. En esta oportunidad presentamos la información correspondiente a la perspectiva de los adolescentes, destacando resultados relevantes de este contexto específico, intentando dar luz en un campo de investigación donde aún resta por hacer.

\section{Materiales y Método}

\section{Participantes}

Se trabajó con 775 alumnos de los Liceos 1 y 2 y de la Escuela Técnica de una ciudad del interior de Uruguay, de edades comprendidas entre los doce y diecinueve años. La distribución de alumnos por centro correspondió a, 341 alumnos del Liceo $\mathrm{N}^{0} 1$ de $1^{\text {er }}$ a $3^{\text {er }}$ año; 302 alumnos del Liceo $\mathrm{N}^{\circ} 2$ de $4^{\circ}$ a $6^{\circ}$ año y 132 alumnos de Escuela Técnica de $1^{\circ}$ a $4^{\circ}$ año. Del total de la muestra, 405 fueron mujeres y 370 varones correspondiendo al $52.2 \%$ y $47.8 \%$ del alumnado consultado. Las edades se encontraban en el rango de 12 a 19 años.

\section{Instrumento}

El instrumento utilizado para el estudio es el "Cuestionario de Evaluación de la Violencia en la Escuela y el Ocio" (CEVEO); este cuestionario, validado en el Programa de Prevención de la Violencia y Lucha contra la Exclusión en la Adolescencia (Díaz-Aguado et al., 2004), evalúa las situaciones de violencia en el contexto escolar y en el ocio. En nuestro estudio se ha consultado únicamente en relación a la convivencia en el centro en los últimos dos meses y se han adaptado algunos términos para la mejor compresión de los alumnos. El instrumento CEVEO, se divide en 7 bloques. El primero de éstos evalúa el grado de satisfacción del adolescente en diferentes lugares y relaciones, por medio de 13 ítems a responder mediante una escala Likert de 7 puntos en la que 1 significa "muy mal" y 7 "muy bien". Los restantes bloques, del 2 al 7 , se responden por medio de una escala Likert de 4 puntos, en que 1 equivale a que "nunca" le ha pasado y 4 a que le ha pasado "muchas veces". El bloque dos, consulta acerca de situaciones vividas como víctima -con 15 ítems-; el tres, consulta sobre situaciones vividas como agresor -con 15 ítems-; el bloque cuatro, sobre situaciones vividas como espectador -15 ítems.-; el quinto, a quiénes recurren los adolescentes para pedir ayuda -7 ítems-; el sexto, percepción del alumno acerca de cómo reaccionan los profesores ante la violencia -7 ítems- $y$ el bloque siete, pregunta sobre cuál ha sido la actitud del alumno ante la agresión entre compañeros, con 6 opciones de respuesta. 


\section{Diseño y Procedimiento}

Se evalúa mediante autoinforme la visión del alumnado. Esta investigación descriptiva permite establecer las frecuencias generales sobre el fenómeno de la violencia escolar, como así también especificar las frecuencias de cada tipo de situación específica consultada.

Se tramitó con los Centros Educativos el consentimiento para la realización del estudio. La administración insumió 40 minutos con cada grupo de estudiantes, previa explicación. Para el procesamiento de los datos recogidos se utilizó el programa SPSS 15.0., con el que se realizó análisis del tipo descriptivo y correlacional.

\section{Resultados}

En relación a la satisfacción y calidad de vida de los adolescentes en los centros educativos, se los consultó acerca de cómo se sentían en diferentes situaciones propuestas. Respondieron de acuerdo a una escala de Likert de 1 a 7 (1 "muy mal" y 7 "muy bien"). Como muestra el Gráfico 1, se encontró en sus respuestas un nivel de satisfacción que oscila entre 5 y 7 . Las puntuaciones mínimas halladas, si bien altas, refieren al centro de estudios; a su relación "con lo que aprenden", "con los profesores" y con "el instituto" siendo ésta la puntuación más baja. La puntuación más alta se presentó en las relaciones con "los amigos" y en segundo lugar "con la madre".

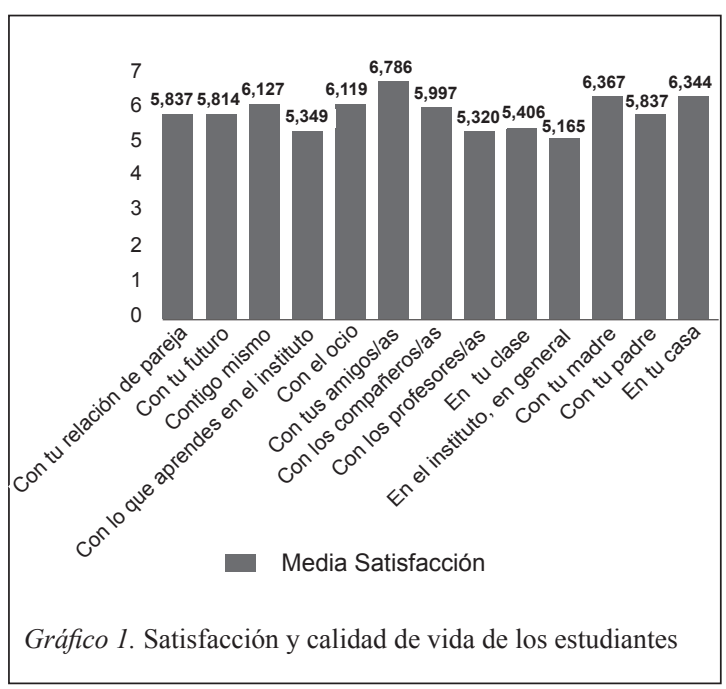

Se resume a continuación, en relación a las respuestas de los estudiantes, la incidencia de las situaciones de violencia escolar vividas por los adolescentes. Los alumnos respondieron a cada ítem, en una escala Likert de 1 a 4 (1 "nunca", 2 "a veces", 3 "a menudo", 4 "muchas veces"). Se indagó acerca de quiénes podrían haber vivido situaciones de violencia desde los roles de víctima, agresor y/o espectador, además de la frecuencia con que han experimentado las diversas formas de violencia escolar.

En los casos de situaciones vividas como víctima de violencia escolar, las respuesta de los alumnos evidenciaron que en las frecuencias de "a veces", "a menudo" o "muchas veces" (reunidas), las situaciones de mayor ocurrencia son las referentes a exclusión social y agresión verbal con situaciones como, "hablan mal de mi" $(42,8)$, "me llaman por sobrenombres..." $(40,09)$, "me insultan" $(37,2)$ y "me ignoran" $(36,6)$. Otras formas de exclusión como, "me rechazan" $(20,2)$ o "me impiden participar" $(24,9)$ aparecen en porcentajes altos, mayores al $20 \%$. Las formas de violencia de gravedad media, entendidas como agresión física indirecta (Defensor del Pueblo, 2007), presentan los siguientes porcentajes: "me esconden cosas" $(36,3)$, "me roban cosas" $(16,9)$ y "me rompen cosas" $(16,7)$. Los porcentajes más bajos fueron hallados en las formas de violencia escolar de mayor gravedad, "me amenazan con armas" $(2,5)$ y "me obligan con amenazas a situaciones de carácter sexual" $(2,7)$, (ver Gráfico 2).

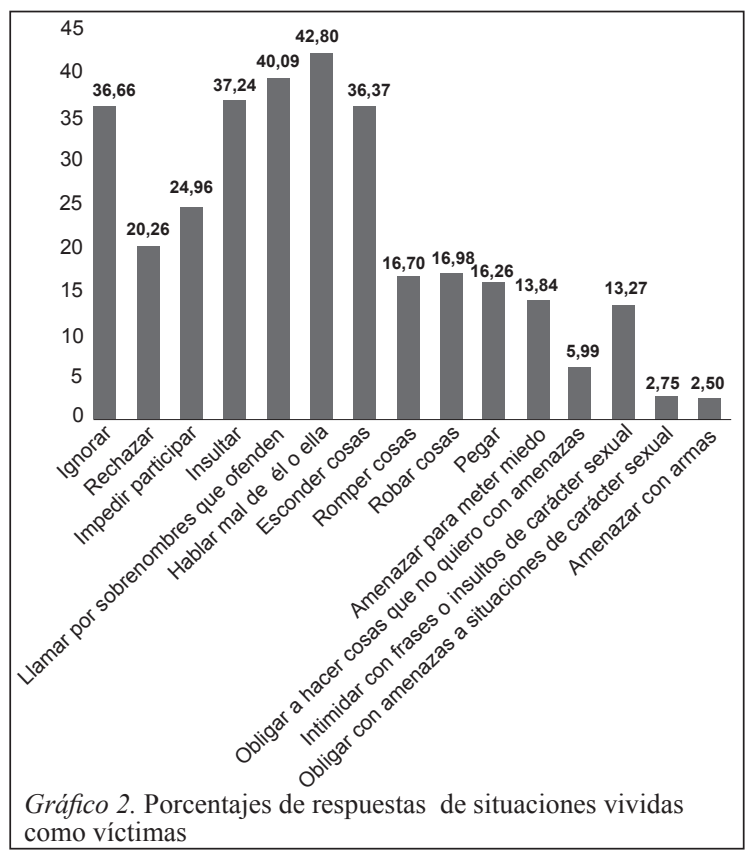


Para facilitar cálculos posteriores, se realizaron análisis factoriales de los bloques de respuestas acerca de situaciones vividas como víctimas, agresores y espectadores. El análisis de componentes principales y con rotación Varimax, los factores que se indican en la Tabla 1 para situaciones de victimización.

Tabla 1. Factores en situaciones de victimización

\begin{tabular}{|c|c|c|}
\hline Factores & Situaciones componentes & $\begin{array}{l}\text { Alfa de } \\
\text { Cronbach }\end{array}$ \\
\hline $\begin{array}{l}\text { 1. Exclusión } \\
\text { social y } \\
\text { agresión verbal }\end{array}$ & $\begin{array}{l}\text { ignorar, insultar, hablar } \\
\text { mal de alguien, impedir } \\
\text { participar, rechazar, llamar } \\
\text { por sobrenombres para } \\
\text { ofender o ridiculizan }\end{array}$ & ,78 \\
\hline $\begin{array}{l}\text { 2. Victimización } \\
\text { de gravedad } \\
\text { media }\end{array}$ & $\begin{array}{l}\text { robar cosas, esconder } \\
\text { cosas o romper cosas }\end{array}$ & ,61 \\
\hline $\begin{array}{l}\text { 3. Victimización } \\
\text { de gravedad } \\
\text { extrema }\end{array}$ & $\begin{array}{c}\text { pegar, amenazar para } \\
\text { meter miedo, obligar a } \\
\text { hacer cosas que no quiere } \\
\text { con amenazas, intimidar } \\
\text { con frases o insultos de } \\
\text { carácter sexual, obligar con } \\
\text { amenazas a situaciones de } \\
\text { carácter sexual, amenazar } \\
\text { con armas }\end{array}$ & ,78 \\
\hline
\end{tabular}

Las correlaciones entre factores fueron relativamente altas, siendo entre el factor $1 \mathrm{y}$ 2 de .420; entre el 1 y 3 de .502 , y la más alta entre los factores 2 y 3 de .542. Con lo cual, se podría hipotetizar que las víctimas de violencia de gravedad media, también lo son de situaciones de violencia de gravedad extrema.

En relación a las situaciones en las que participó como agresor, se consultó a los estudiantes acerca de si habían participado en determinadas situaciones molestando a algún compañero en el instituto, en los últimos dos meses. Los porcentajes de respuestas de los alumnos se resumen en el Gráfico 3.

Los alumnos principalmente agreden "poniendo sobrenombres que ofenden..." $(45,5)$, "hablando mal de él o ella" $(43,6)$, "insultando" $(37,8)$, "ignorando" $(36,9)$ y "rechazando" $(28,7)$, es decir excluyendo y con agresiones verbales. Cabe destacar, que si bien estas situaciones también han sido señaladas por las víctimas como las de mayor frecuencia, en el caso de los agresores, los porcentajes resultan aún mayores. Ocurre lo contrario con situaciones como "escondiendo cosas" (24) e "impidiendo participar" $(14,7)$. En ambas, las víctimas han señalado porcentajes superiores

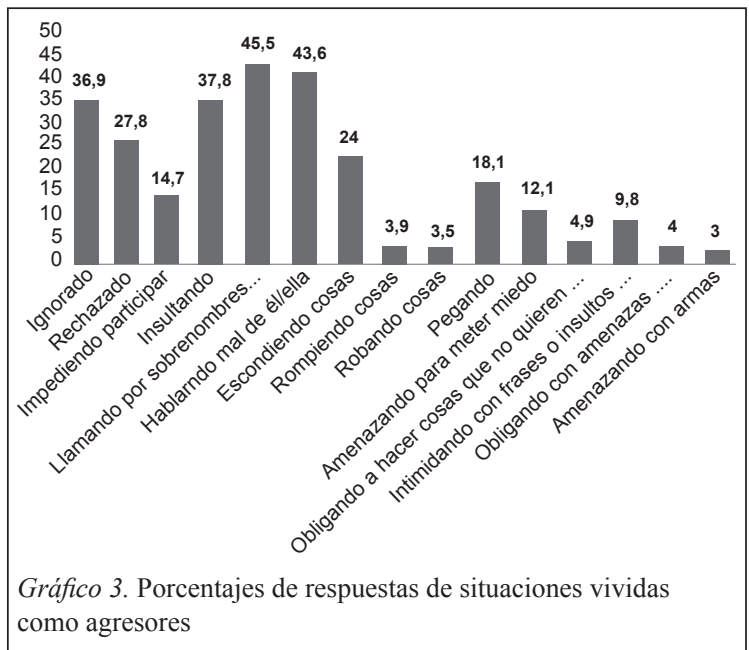

a los señalados por los agresores. En el resto de situaciones, entendidas como de mayor gravedad, los porcentajes de ocurrencia son menores, rondando el $4 \%$. Se destacan entre ellas, "pegando" $(18,1)$, "amenazando para meter miedo" $(12,1)$ e "intimidando con frases o insultos de carácter sexual" $(9,8)$.

Se obtuvieron dos factores en el análisis de componentes principales y rotación Varimax, para las situaciones vividas como agresores. Los mismos se presentan en la Tabla 2.

Tabla 2. Factores en situaciones de agresión

\begin{tabular}{ccc}
\hline Factores & Situaciones componentes & $\begin{array}{c}\text { Alfa de } \\
\text { Cronbach }\end{array}$ \\
\hline $\begin{array}{c}\text { 1. Exclusión } \\
\text { social y } \\
\text { agresión } \\
\text { de gravedad } \\
\text { media }\end{array}$ & $\begin{array}{c}\text { ignorando, rechazando, impidiendo } \\
\text { participar, insultando, poniendo } \\
\text { sobrenombres que le ofenden y } \\
\text { ridiculizan, hablando mal de él o } \\
\text { ella, escondiendo cosas }\end{array}$ \\
& $\begin{array}{c}\text { rompiendo cosas, robando cosas, } \\
\text { pegando, amenazando para meter } \\
\text { miedo, obligando a hacer cosas } \\
\text { quresión } \\
\text { grave }\end{array}$ & $\begin{array}{c}\text { que no quiere con amenazas, } \\
\text { inimidando con frases o insultos } \\
\text { de carácter sexual, obligando } \\
\text { con amenazas a conductas o } \\
\text { situaciones de carácter sexual } \\
\text { de las que no quiere participar, } \\
\text { amenazando con armas }\end{array}$ \\
\hline
\end{tabular}

La correlación entre ambos factores es de .640 , puntuación relativamente alta que permitiría hipotetizar que, aquellos alumnos que agreden en situaciones de exclusión social y agresiones de gravedad media, también lo hacen en situaciones de agresión de gravedad.

Como se aprecia en el material que antecede, todas las situaciones de violencia escolar 
consultadas a los estudiantes, existen y ocurren en el espacio educativo o al menos así ha sucedido en los últimos dos meses de convivencia.

A continuación se presentan los porcentajes de respuesta en relación a situaciones que los estudiantes han conocido sin participar directamente, es decir como espectador. La tendencia de respuestas se corresponde a la presentada por las víctimas y los agresores, pero con porcentajes mayores. Las situaciones de mayor ocurrencia señaladas por los espectadores con una frecuencia de "a veces", "a menudo" y "muchas veces", refieren a situaciones de exclusión social y agresiones verbales, y son el "poner sobrenombres que ofenden o ridiculizan" $(72,2)$, "hablar mal de él o ella" $(68,8)$ e "insultar" $(65,5)$. Los porcentajes más reducidos, los encontramos en las situaciones de violencia de gravedad extrema como son "obligar a hacer cosas que no quiere con amenazas" $(20,8)$, "obligar con amenazas a situaciones de carácter sexual" $(10,85)$ y "amenazar con armas" $(9,9)$. Como en el caso de víctimas y agresores, en los espectadores, al aumentar la frecuencia de ocurrencia de las situaciones propuestas, los porcentajes se reducen.

El análisis de componentes principales y la rotación Varimax, permitió hallar dos factores, que

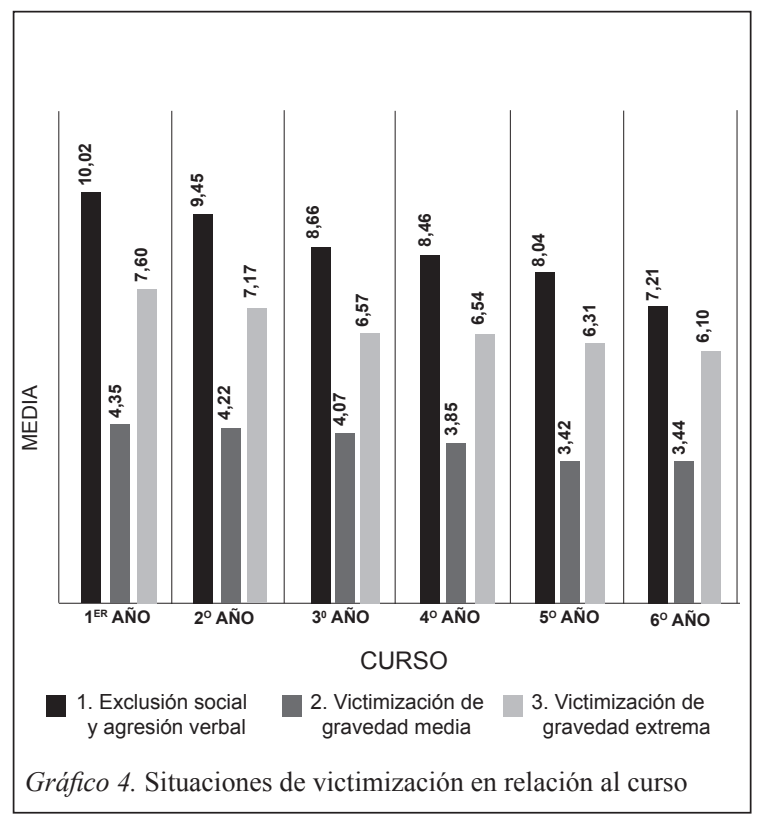

se corresponden a nivel de situaciones que los componen, a los hallados en el caso de los agresores. Se los llamó: Exclusión social y Agresión de gravedad media y al factor dos, Agresión grave.

Con el objetivo de analizar la incidencia de la violencia escolar en relación al curso, se realizaron análisis descriptivos que evidenciaron la relación con los factores asociados a los roles de víctima, agresor y espectador. Además, las pruebas de homogeneidad de la varianza y ANOVA pusieron de relieve la no homogeneidad entre factores, es decir, la diferencia significativa entre los mismos, también para los tres roles. Los análisis evidenciaron que en el caso de las víctimas y para los tres factores, las situaciones de violencia ocurren principalmente en primer año, disminuyendo hacia sexto año (ver Gráfico 4).

Para los agresores, en el factor de exclusión social y agresión de gravedad media, la mayor ocurrencia la encontramos en segundo año, siguiendo el orden descendente hacia tercer año y primer año. En cuarto, quinto y sexto año, los valores descienden aún más. En el caso del factor de agresión grave, el máximo de ocurrencia lo encontramos en tercer año, descendiendo en segundo y posteriormente en primero. Continuando hacia cuarto, quinto y sexto año, los porcentajes se reducen en este orden (ver Gráfico 5).

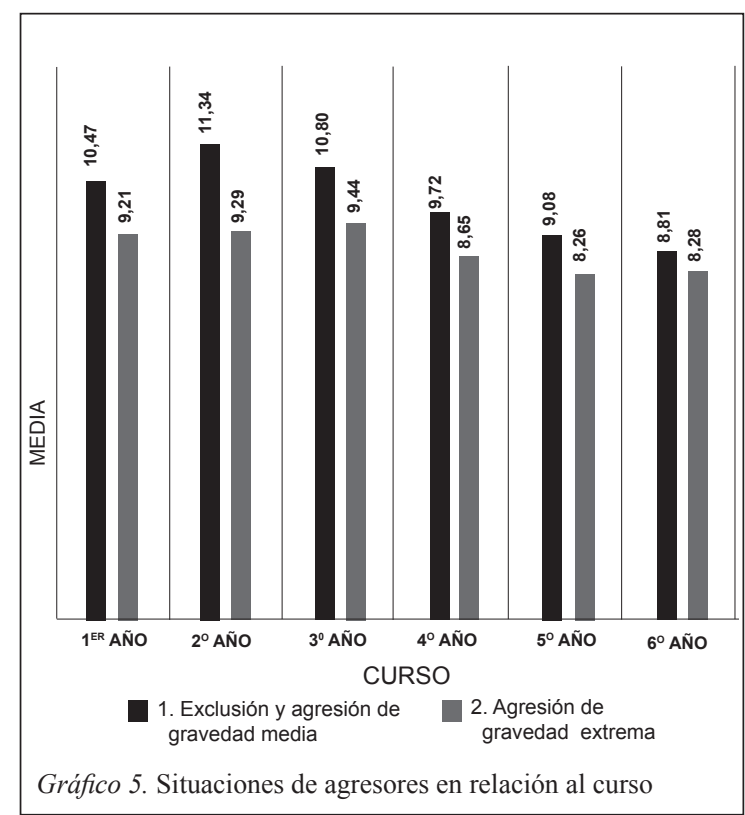


Finalmente, para los espectadores, el máximo de ocurrencia se presenta en primer año y desciende paulatinamente hacia sexto año, en ambos factores (ver Gráfico 6). Se encontró que los máximos porcentajes en primer año, tanto para las víctimas como para los espectadores de la violencia escolar se concentraron en exclusión y agresión de gravedad media. Lo que se reitera en el mismo factor en el segundo curso; en tanto en el tercer curso los máximos se dan en el factor agresión extrema.

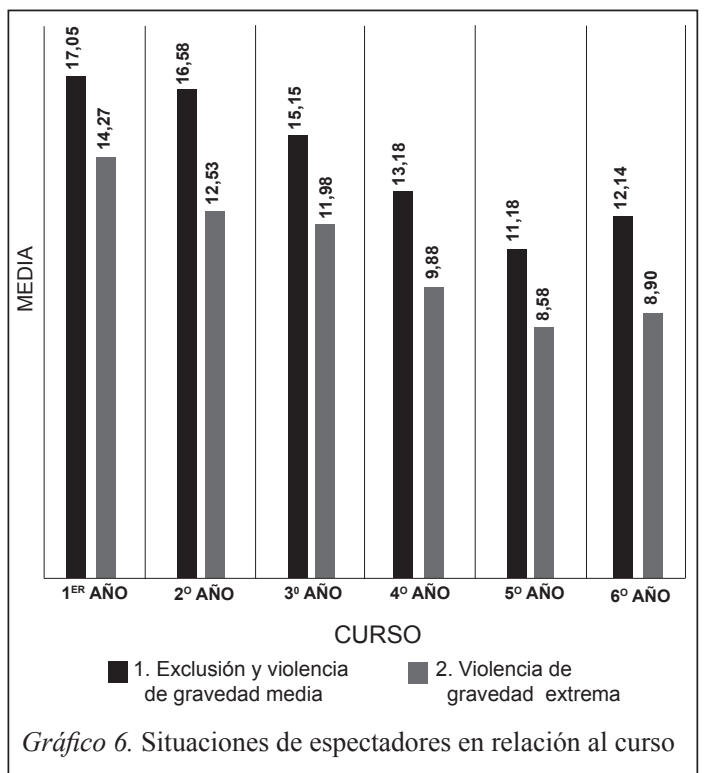

Se ha consultado sobre qué hacen los adolescentes frente a la agresión a un compañero. Los alumnos respondieron con una frecuencia de "a menudo" o "muchas veces" que "intentan cortar la situación si es un amigo" $(74,1)$.

En segundo lugar han señalado la opción propuesta de "intento cortar la situación aunque no sea un amigo" $(46,2)$, seguido de "pido ayuda a un profesor" $(27,5)$, "me meto con él lo mismo que el grupo" $(22,1)$, "no hago nada aunque debería hacerlo" $(21,2)$ o, "no hago nada, no es mi problema" $(16,3)$. Si bien las tres últimas opciones son las de menor porcentaje, resultan relevantes y con porcentajes a considerar -rondan el $20 \%$-, en tanto que se podrían entender como acciones de complicidad ante la violencia escolar, particularmente en el caso de "me meto con él lo mismo que el grupo".

Otro objetivo de la investigación fue conocer los recursos con que cuentan los adolescentes en situaciones de violencia escolar, es decir, a quiénes pueden recurrir, quiénes les apoyan ante situaciones vividas directamente o conocidas indirectamente. Como figuras disponibles a ayudar cuando han sufrido una agresión ellos mismos, con una frecuencia de "a menudo" o "muchas veces" los alumnos dicen recurrir en primer lugar a "los amigos" $(73,4)$, en segundo lugar a la "madre" $(70,4)$ y luego al "padre" $(62,8)$. Los porcentajes disminuyen a niveles de ocurrencia semejantes, cuando señalan como figuras de apoyo y a las cuáles pueden recurrir al "tutor/ orientador" $(46,4)$, a "los compañeros" $(45,4)$, "a otros" $(43,4)$ y en último lugar encontramos que recurren a "los profesores" $(43,1)$. En tanto que en el caso de que el agredido sea un/a compañero/a, los alumnos recurren también en primer lugar a "los amigos" $(69,5)$ y en segundo lugar a "la madre" (58), aunque los porcentajes son menores que cuando la agresión ha sido vivida por ellos mismos. Recurrir a "los profesores" como figura de apoyo es, una vez más, algo que ocurre en menor medida, en este caso el porcentaje último, posterior a "los profesores" $(46,9)$, lo lleva "otros" $(39,7)$. La Tabla 3 reúne los resultados aquí mencionados.

Tabla 3. Personas a las que recurren los estudiantes cuando son agredidos y cuando compañeros/as son agredidos

\begin{tabular}{lccccccc}
\hline & Amigos/as & $\begin{array}{c}\text { Compañeros } \\
\text { /as }\end{array}$ & $\begin{array}{c}\text { Tutor/a } \\
\text { orientador/a }\end{array}$ & Profesores/as & Padre & Madre & Otros \\
\hline $\begin{array}{l}\text { Cuando él/ella es } \\
\text { agredido de forma } \\
\text { directa }\end{array}$ & 73,4 & 45,4 & 46,4 & 43,1 & 62,8 & 70,4 & 43,4 \\
$\begin{array}{l}\text { Cuando un } \\
\text { compañero/a } \\
\text { es agredido }\end{array}$ & 69,5 & 56,7 & 51,1 & 46,9 & 54,6 & 58,0 & 39,7 \\
\hline
\end{tabular}


Se indagó además, la perspectiva de los alumnos acerca de qué hace el profesorado en situaciones de violencia. Los adolescentes respondieron, con una frecuencia de "a menudo" o "muchas veces", que "podemos contar con algún profesor cuando alguien intenta abusar" $(62,5)$, siendo éste el porcentaje más alto de ocurrencia entre las situaciones propuestas. Le siguen en descenso las respuestas que señalan que los profesores "intervienen para cortar la situación" (57,8), "actúan como mediadores" (55) y "trabajan activamente para prevenir" $(50,3)$. A continuación, los porcentajes corresponden a las situaciones entendidas como más negativas en cuanto a la perspectiva de los alumnos acerca de los profesores, siendo éstas "no saben impedirlo" $(33,1)$, "no se enteran" $(30,8)$ o "miran para otro lado" $(19,9)$.

Finalmente y acorde a los objetivos de la investigación macro desarrollada (Baridon, 2010), se analizaron las situaciones de riesgo entre los estudiantes, intentando identificar posibles casos sospechosos de riesgo de bullying a confirmar en análisis posteriores. Siguiendo las "normas de interpretación de datos" de Díaz- Aguado et al. (2004, p. 65), se realizaron los estadísticos descriptivos de cada uno de los factores de las situaciones de víctimas y agresores; así como histogramas para cada factor, lo cual permitió conocer la distribución de frecuencias y se seleccionaron los percentiles 95 como corte para las dimensiones de la violencia escolar evaluadas. La Tabla 4 a continuación resume los resultados hallados. Con lo cual para las situaciones de victimización se encontró que un 3,2 \% de alumnos que han vivido situaciones de exclusión social y agresión verbal, que un $3,5 \%$ de alumnos que han vivido situaciones de gravedad media y un $3,5 \%$ del alumnado que ha vivido situaciones de gravedad extrema, podrían encontrarse en situaciones de riesgo y ser -en análisis futurosconsiderados como posibles víctimas de bullying. En tanto para las situaciones de agresión, se encontró que un $3.9 \%$ de los alumnos que participan como agresores en situaciones de exclusión y gravedad media y un $4,6 \%$ de los alumnos agresores en situaciones de gravedad extrema, podrían encontrarse en situaciones de riesgo y ser, con análisis posteriores, considerados como posibles agresores, en situaciones de bullying.

\section{Discusión}

Los estudiantes consultados han valorado en general de forma positiva su calidad de vida en las situaciones y contextos propuestos, siendo la media de todas las situaciones valoradas entre 5 y 7 , en tanto 7 fue el valor de mayor satisfacción. Desde el punto de vista teórico (Coleman \& Hendry, 2003), existe una diferencia entre la calidad y valoración de las relaciones con los amigos y con los compañeros, aspecto que se ha visto reflejado en los resultados y que ya se ha presentado en otros contextos de investigación (Coleman \& Hendry, 2003; DíazAguado et al., 2004). La relación con amigos suele ser más estrecha que con compañeros, diferencia que podría verse incrementada si algunos estudiantes no se sienten cómodos en el grupo de clase y su percepción del mismo es negativa (O'Brien \& Bierman, 1988), siendo la no reciprocidad en las relaciones "un fuerte predictor de la victimización" (Lisboa \& Koller, 2009, p. 174). Este aspecto concuerda con la necesidad de intervenir para potenciar las relaciones entre compañeros de clase y crear un espacio educativo que promueva el desarrollo de "las competencias necesarias para relacionarse pacíficamente con los demás" (como se citó en Chaux \& Velásquez, 2008, p. 32).

Las altas puntuaciones encontradas en el relacionamiento con "la madre" y también con "el padre", a la hora de considerar una figura de ayuda disponible ante las situaciones de violencia escolar, ya sean los propios adolescentes como víctimas o si ésta se trata

Tabla 4. Estadisticos para los factores de victimización y agresiones

\begin{tabular}{lccccc}
\hline \multicolumn{1}{c}{ Estadísticos } & \multicolumn{5}{c}{ Factores } \\
\hline & $\begin{array}{c}\text { Exclusión social y y } \\
\text { agresión verbal }\end{array}$ & $\begin{array}{l}\text { Victimización de } \\
\text { gravedad media }\end{array}$ & $\begin{array}{c}\text { Victimización de } \\
\text { gravedad extrema }\end{array}$ & $\begin{array}{c}\text { Exclusión y } \\
\text { agresión de } \\
\text { gravedad media }\end{array}$ & $\begin{array}{c}\text { Agresión de } \\
\text { gravedad } \\
\text { extrema }\end{array}$ \\
\hline Media & 8,95 & 4,00 & 6,81 & 10,21 & 8,94 \\
Desv. típica & 3,27 & 1,54 & 2,06 & 3,76 & 2,83 \\
Percentiles 95 & 16 & 7 & 10 & 18 & 13,45 \\
\% sobre el percentil & 3,2 & 3,5 & 3,5 & 3,9 & 4,6 \\
\hline
\end{tabular}


de un compañero, coinciden con resultados de otras investigaciones (Díaz-Aguado et al., 2004). Esto muestra la importancia que presenta en el desarrollo del niño y del adolescente la relación con su familia. Lo cual pone indirectamente en primer plano la necesidad de potenciar la relación entre profesorado y centro educativo en general, con los padres y madres de los alumnos, como una vía principal para conseguir una intervención eficaz en la detección y prevención de la violencia escolar. Son varios los autores que sitúan su mirada en esta necesidad de reflexión y actuación de cara a potenciar la relación escuela-familia (Martínez Cerón, 2005, González, 2006; López López, 2006). Relación que permitiría generar nuevos espacios de comunicación y coordinación de cara a trabajar en la erradicación de la violencia escolar.

Por otra parte, las puntuaciones bajas en las respuestas del alumnado las encontramos en sus relaciones con "el instituto", "los docentes" y "lo que aprenden". A simple vista aquí hallamos un problema a la vez que una paradoja. Alumnos y docentes conviven cotidianamente en el espacio del instituto y a nivel de calidad de vida, esta relación es la que presenta menores puntuaciones. Podríamos asociar esta información a la percepción manifestada por una importante parte de los alumnos, en cuanto a lo que hacen los docentes ante la violencia escolar; recordemos que entre un $20-30 \%$ señalaron que los profesores "no saben impedirlo", "no se enteran" o "miran para otro lado". Además los estudiantes dicen acudir a ellos ante la violencia escolar, como último o penúltimo recurso. Otra investigación también realizada en Uruguay (Giorgi et al., 2012) señala que los adolescentes critican "la pasividad docente" (p. 85) ante la violencia escolar, reclaman mayores actuaciones de cara a "parar las situaciones a tiempo", poner límites. Son los propios estudiantes, los que reclaman cambios en la relación alumno-profesor, aspecto manifestado en particular por los estudiantes consultados, como así en el contexto español (Díaz-Aguado et al., 2004; Defensor del Pueblo, 2007). Dado lo relevante de esta información, es importante profundizar en el estudio de las relaciones entre alumnos y docentes, a la vez que promover una reflexión y autocrítica a nivel del colectivo de profesores.

Se encontró en todos los centros y grupos de estudiantes consultados, la existencia de situaciones de violencia escolar en las que el alumnado desempeña diferentes roles de vícti- mas, agresores o espectadores de la violencia. Aspecto que concuerda con las teorías comentadas inicialmente acerca de la ubicuidad de la violencia (Torrego \& Moreno, 2003) y de sus diferentes expresiones en los diversos ámbitos (Giorgi et al., 2012). Este trabajo deja abierta la necesidad de profundizar en el conocimiento y dinámicas de interacción de este colectivo de estudiantes de los centros. Asimismo, el haber identificado mayores índices de ocurrencia en situaciones de violencia de menor gravedad como son las de exclusión social y agresión verbal, no reduce su importancia. Por el contrario, afectan a quién las vive y perpetuadas en el tiempo, dañan en gran medida a los adolescentes (Hugh-Jones \& Smith, 1999), siendo la exclusión, según Lisboa y Koller (2009), un predictor relevante de la victimización de los adolescentes.

En estudios llevados a cabo en centros escolares de la Comunidad Autónoma de Madrid en España (Defensor del Pueblo, 2007; DíazAguado et al.,2004), también se ha encontrado que las situaciones de violencia escolar menos graves (rechazo verbal y exclusión pasiva) son las más frecuentes, seguidas por la violencia verbal y aquella que se ejerce sobre las propiedades. Asimismo, estos estudios pusieron de manifiesto que estas situaciones de violencia no eran episodios aislados sino que formaban parte de la vida cotidiana de los centros escolares madrileños, dando lugar a situaciones repetitivas tanto para las víctimas como para los agresores y espectadores de la violencia escolar.

La estabilidad que suele darse en los roles de víctima y agresor (Olweus, 2004), resulta fundamental en la consolidación de la violencia escolar, con lo cual además de la defensa que hacemos en nuestra investigación al respecto de la intervención a nivel de grupo, proponemos el tratamiento cercano y particular de cada caso.

En base a las ideas ya propuestas por Olweus (2004), entendemos al colectivo de espectadores de la violencia escolar, como un grupo que puede llegar a ser determinante de cara a erradicar la violencia escolar. Olweus (2004) propone que los alumnos espectadores, viven las situaciones de violencia identificándose desde variados roles: algunos apoyan activamente al agresor, otros apoyan al agresor pero no activamente, otros apoyan a la víctima pero no hacen nada para frenar la situación y otros son los defensores directos de la víctima. En esta investigación se encontró un alto porcentaje de alumnos espectadores de la violencia y que 
además, muchas veces apoyan directa o indirectamente a los agresores. Internacionalmente existen investigaciones (Smith, 1998; Torrego, 2006; Díaz-Aguado, 2009), que destacan la importancia de la actitud del espectador frente a la violencia. Desde nuestra visión en particular, se entiende que se podría trabajar con este grupo de alumnos, enfocando su rol hacia la mediación, la negociación, a tomar posición en la intolerancia a la violencia, colaborando en disminuir estas situaciones. Sería adecuado para ello profundizar en las dinámicas de socialización de los grupos de alumnos, en la red de vínculos que se generan entre adolescentes y en las conductas aceptadas a nivel de grupo (Viscardi, 2004; Blanco, Caballero, \& De la Corte, 2007; Berger, 2009). Un análisis de estos aspectos, resultaría un buen punto de partida de un trabajo desde y con los adolescentes hacia la erradicación de la violencia escolar.

Es necesario intervenir en esta tarea de construcción de la no violencia y dependemos del compromiso docente, pieza fundamental al trazar objetivos en esta área. Así como también de quienes puedan otorgarles a estos, formación, herramientas y recursos para trabajar en contra de la violencia. Hay evidencia de lo relevantes que resultan las intervenciones destinadas a mejorar la convivencia atendiendo a estos aspectos (Torrego, 2006) y entendemos que es posible de desarrollar en este contexto específico, el cual ha evidenciado en el desarrollo de la investigación macro, interés en el tema.

La investigación desarrollada en este contexto específico, ha permitido confirmar la existencia de diversas formas de violencia escolar, vividas en todos los grupos de los diferentes centros educativos participantes, así como una percepción un tanto negativa de la postura del profesorado al enfrentar esta violencia escolar, por parte de los adolescentes.

Este trabajo abre las puertas a nuevas líneas de investigación en este ámbito relacionadas a los aspectos antes comentados, a decir, profundización en el conocimiento de las relaciones a nivel de grupos; profundización en el conocimiento de las relaciones entre estudiantes y profesores; promoción de estrategias de comunicación entre centros educativos y familias; desarrollo de un programa de intervención que comprometa al alumnado así como a docentes, en la construcción de un espacio de convivencia de calidad para todos. Entendemos que de alguna forma, el propio hecho de investigar en los centros participantes del estudio, ha sido y es punto de partida de reflexión de gran parte de los estudiantes y docentes consultados.

La autocrítica realizada ha permitido evidenciar algunas limitaciones como las que surgen de no poder contar con datos de un estudio más amplio, a la vez que, uno del tipo longitudinal; así como, conocer sobre las dinámicas que se desarrollan en el contexto de las redes sociales en cuanto a la violencia escolar y su incidencia en el sistema. No obstante posibilitó un acercamiento a la realidad de los centros educativos en cuanto a la violencia escolar y desde roles diferenciados. Todo lo anterior supone una instancia de reflexión y un desafío.

\section{Referencias}

Baridon, D. (2010) Estudio de la violencia escolar en los centros de Educación Media de Juan Lacaze, Uruguay. (Tesis Doctoral). Universidad de Alcalá, España.

Berger, C. (2009) ¿Es la agresividad adaptativa? Evidencias de su función social en adolescentes chilenos. En C. Berger y C. Lisboa (Eds.) Violencia Escolar. Estudios y posibilidades de intervención en Latinoamérica ( $\mathrm{p}$. 139-160). (1 ${ }^{\mathrm{a}}$. ed). Santiago de Chile: Universitaria.

Blanco, A., Caballero, A., \& De la Corte, L. (2007) Psicología de los grupos. Madrid: Pearson Educación.

Bronfenbrenner, U. (1987) La ecología del desarrollo humano. (1ª. ed.). Barcelona: Paidós.

Cajigas, N., Kahan, E., Luzardo, M., Ugo, M., Najson, S. , \& Zamalvide, G. (2006). Agresión entre pares (bullying) en un centro educativo de Montevideo: Estudio de las frecuencias de los estudiantes de mayor riesgo. Revista Médica del Uruguay, 22, 143-151.

Cajigas, N., Luzardo, M., \& Mungay, M. (2013). Una mirada psicológica de la violencia en los centros educativos. Prevalencia de afectos, actitudes y conductas negativas de adolescentes del secundario público en Montevideo, Uruguay. Deutschland, Alemania: Académica Española.

Cajigas, N., Luzardo, M., \& Ugo, M. (2009) ¿Es la depresión la contracara de la agresión? Un estudio en estudiantes uruguayos de enseñanza media. En C. Berger y C. Lisboa (Eds.) Violencia Escolar. Estudios y posibilidades de intervención en Latinoamérica. (pp. 183-209) ( $1^{\text {a }}$ ed.). Santiago de Chile: Universitaria.

Chaux, E., \& Velásquez, A. M. (2008) Violencia en los colegios de Bogotá: contraste internacional y algunas recomendaciones. Revista Colombiana de Educación, 55, 14-37.

Coie, J. D., \& Dodge, K. A. (1998) Aggression and antisocial behavior. En W. Damon y N. Eisenberg (Eds.) Handbook of Child Psichology. Volume 3. Social, emotional and personality development. New York: Wiley \& sons.

Coleman, J. C., \& Hendry, L. B. (2003) Psicología de la adolescencia. Madrid: Morata.

Defensor del Pueblo (2007) Violencia Escolar: El maltrato entre iguales en la Educación Secundaria Obligatoria 1999-2006. (Nuevo Estudio y actualización del Informe 2000). Madrid: Publicaciones de la Oficina del Defensor del Pueblo.

Del Barrio, C., Martín, E., Almeida, A., \& Barrios, A. (2003) Del maltrato y otros conceptos relacionados con la agresión entre escolares, y su estudio psicológico. Infancia y Aprendizaje, 26 (1), 9-24. 
Díaz-Aguado, M. J. (2004) Prevención de la violencia y lucha contra la exclusión desde la adolescencia. La violencia entre iguales en la escuela y el ocio. Tomo II. Programa de intervención y estudio experimental. Madrid: Instituto de la Juventud.

Díaz-Aguado, M. J. (2009) Ayudando a resolver conflictos. Convivencia escolar y prevención de la violencia. Recuperado de: http://ntic.educacion.es/w3/recursos2/ convivencia escolar/

Díaz-Aguado, M. J., Martínez, R., \& Martín, G. (2004) Prevención de la violencia y lucha contra la exclusión desde la adolescencia. La violencia entre iguales en la escuela y el ocio. Tomo I. Estudios comparativos e instrumentos de evaluación. Madrid: Instituto de la Juventud.

Fernández, I. (1998) Prevención de la violencia y resolución de conflictos. El clima escolar como factor de calidad. Madrid: Narcea.

Galtung, J. (1998) Tras la violencia, 3 R: reconstrucción, reconciliación, resolución. Bilbao: Gernika Gogoratuz.

Giorgi, V., Kaplún, G., \& Morás, L. E. (2012) La violencia está en los otros. La palabra de los actores educativos. Montevideo: Trilce.

González, M. (2006) Familias y centros educativos. Buscando puntos de encuentro frente al hostigamiento escolar. Recuperado de http://www.ucu.edu.uy/Facultades/Psicologia/TB/Documentos/Familias_y_centros_educativos.pdf

Hugh-Jones, S., \& Smith, P.K. (1999) Self-reports of short - and long-term effects of bullying on children who stammer. British Journal of Educational Psichology, 69,141-158.

Hurrelman, K. (1990) Wie kommt es zu Gewalt in der Schule und was konnen wir dagegen tun? Kind, Jugend und Gesellschaft, 36, (4), 103-108.

Kornblit, A.; Adaszko, D., \& Di Leo, F. (2009) Clima social escolar y violencia: un vínculo explicativo posible. Un estudio en escuelas medias argentinas. En C. Berger y C. Lisboa (Eds.) Violencia Escolar. Estudios y posibilidades de intervención en Latinoamérica ( $\mathrm{pp}$. 109-137). ( $1^{\text {a }}$ ed.). Santiago de Chile: Universitaria.

Lisboa, C., \& Koller, S. (2009) Factores protectores y de riesgo para la agresividad y victimización en escolares brasileños: el rol de los amigos. En C. Berger y C. Lisboa (Eds.) Violencia Escolar. Estudios y posibilidades de intervención en Latinoamérica (pp. 161-181). (1 ${ }^{\mathrm{a}}$. Ed.). Santiago de Chile: Universitaria.

López López, M. T. (2006) La familia en el proceso educativo. Madrid: Cinca.

Martín, G. (2008) La mediación como herramienta de prevención de la violencia escolar. Revista de Mediación,1, 26-31.

Martínez Cerón, G. (2005) Sombras y luces de la relación familia y escuela. En J. M. Escudero Muñoz, A. Guarro Pallás \& G. Martínez, Sistema educativo y democracia: alternativas para un sistema escolar democrático (pp. 99-150). Barcelona: Octaedro

Martínez-Otero, V. (2005) Conflictividad escolar y fomento de la convivencia. Revista Iberoamericana de Educación, 38, 33-52.
O'Brien, S. F., \& Bierman, K. L. (1988) Conceptions and perceived influence of peer groups: interviews whit preadolescents and adolescents. Child Development, $59,1360-1365$.

Olweus, D. (2004) Conductas de acoso y amenazas entre adolescentes. Madrid: Morata.

Ortega, R., Del Rey, R., \& Mora-Merchán, J. (2001) Violencia entre escolares: Conceptos y etiquetas verbales que definen el fenómeno de maltrato entre iguales. Revista Interuniversitaria de Formación del Profesorado, 41, 95-113.

Parke, R. D., \& Slaby, R. G. (1983) The development of aggression. En P. H. Mussen (Ed). Handbook of Child Psychology, Vol. 4.Socialization, personality, and social development (pp. 547-641). New York: Wile+y \& sons

Pellegrini, A. D. (2002) Bullying, victimization and sexual harassment during the transition to Middle School. Educational Psychologist, 37(3), 151-163.

Pérez Algorta, G. (2006) Hostigamiento escolar. Violencia de bajo ruido. Recuperado de: http://www.ucu.edu.uy/ Facultades/Psicologia/TB/Documentos/violencia_a_ bajo_ruido.pdf

Pikas, A. (2002) New Developments of the Shared Concern Method. School Psychology Internacional, 23 (3), 307-326.

Pulido, R., Martín, G., \& Lucas, B. (2011) La modalidad de agrupamiento educativo como variable relevante en el análisis de la violencia escolar. Revista de Educación 356, 457-481.

Salmivalli, C.; Lagerspetz, K.; Björkqvist, K.; Österman, K., \& Kaukianen, A. (1996) Bullying as a group process: Participant role and their relation to social status within the group. Aggressive behavior, 22, 1-15.

Shapiro, J. (2006). Youth violence: the Problem and its Potential Solutions. Recuperado de: http://www. ucu.edu.uy/Facultades/Psicologia/TB/Documentos/ Article_for_Uruguay.pdf

Smith, P. K. (1998) El proyecto Sheffield. No sufráis en silencio. Cuadernos de Pedagogía, 270, 51-54.

Suton, J.; Smith, P. K., \& Swettenham, J. (1999) Social cognition and bullying: Social inadequacy or skilled manipulation? British Journal of Developmental Psychology, 17, 435-450.

Torrego, J. C. (coord.) (2006) Modelo integrado de gestión de la convivencia. Estrategias de mediación y tratamiento de conflictos. Barcelona: Graó.

Torrego, J. C. (2007) El modelo integrado: un nuevo marco de educativo para la gestión de los conflictos de convivencia desde una perspectiva de centro. Idea La ManchaRevista de Educación de Castilla-La Mancha, 4, 84-89.

Torrego, J. C., \& Moreno, J. M. (2003) Convivencia y disciplina en la escuela. El aprendizaje de la democracia. Madrid: Alianza.

Viscardi, N. (2004) Conflicto escolar, práctica educativa e identidades juveniles. Trabajo presentado en la conferencia Internacional de Sociología de la Educación: Globalización, educación, resistencia y tecnologías: La responsabilidad social de la sociología de la educación frente a los movimientos sociales emergentes. Universidad de Buenos Aires, Argentina.

Para citar este artículo:

Baridon Chauvie, D., \& Martín Seoane, G. (2014). Violencia escolar en estudiantes de educación media. Ciencias Psicológicas VIII (2): 173 - 183 